\title{
INFLUENCE OF ENDOCARDIAL-EPICARDIAL CROSSOVER OF MUSCLE FIBERS ON LEFT VENTRICULAR WALL MECHANICS
}

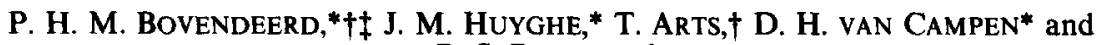 \\ R. S. RENEMAN§ \\ *Department of Mechanical Engineering, Eindhoven University of Technology, Eindhoven, The \\ Netherlands; and +Departments of Biophysics and \$Physiology, Cardiovascular Research Institute \\ Maastricht, University of Limburg, Maastricht, The Netherlands
}

\begin{abstract}
The influence of variations of fiber direction on the distribution of stress and strain in the left ventricular wall was investigated using a finite element model to simulate the mechanics of the left ventricle. The commonly modelled helix fiber angle was defined as the angle between the local circumferential direction and the projection of the fiber path on the plane perpendicular to the local radial direction. In the present study, an additional angle, the transverse fiber angle, was used to model the continuous course of the muscle fibers between the inner and the outer layers of the ventricular wall. This angle was defined as the angle between the circumferential direction and the projection of the fiber path on the plane perpendicular to the local longitudinal direction.

First, a reference simulation of left ventricular mechanics during a cardiac cycle was performed, in which the transverse angle was set to zero. Next, we performed two simulations in which the spatial distribution of either the transverse or the helix angle was varied with respect to the reference situation, the spatially averaged variations being about 3 and $14^{\circ}$, respectively. The changes in fiber orientation hardly affected the pressure-volume relation of the ventricle, but significantly affected the spatial distribution of active muscle fiber stress (up to $50 \%$ change) and sarcomere length (up to $0.1 \mu \mathrm{m}$ change). In the basal and apical region of the wall, shear deformation in the circumferential-radial plane was significantly reduced by introduction of a nonzero transverse angle. Thus, the loading of the passive tissue may be reduced by the endocardial-epicardial crossover of the muscle fibers.
\end{abstract}

\section{INTRODUCTION}

Our understanding of the relation between global and local mechanics of the left ventricle is incomplete. Global left ventricular performance may be determined experimentally by the measurement of cavity pressure and volume. However, experimental assessment of local ventricular mechanics is difficult. Local deformation can be accurately determined simultaneously only at a limited number of sites in the ventricular wall, by measuring the motion of markers attached to the wall (Prinzen et al., 1986; Waldman et al., 1985). Stresses cannot be measured reliably, because insertion of a force transducer damages the tissue (Huisman et al., 1980).

Because of the experimental limitations, mathematical models have been developed in helping to understand left ventricular mechanics (Arts et al., 1982; Beyar and Sideman, 1984; Bovendeerd et al., 1992; Horowitz et al., 1986; Huyghe et al., 1992). In these models, the muscle fibers in the wall were assumed to be parallel to the endocardial and epicardial surfaces. Then the muscle fiber direction may be quantified by the helix angle $\alpha_{\text {helix }}$ (Fig. 1), defined as the angle

Received in final form 5 September 1993.

$\ddagger$ Author to whom correspondence should be addressed.

This study was supported by the Netherlands Organization for Scientific Research NWO, grant No. 900-536-024. between the fiber direction and the local circumferential direction (Streeter, 1979). The transmural distribution of $\alpha_{\text {nelix }}$ is an important determinant of the transmural distribution of ventricular wall stress (Arts et al., 1982; Huyghe et al., 1992; Bovendeerd et al., 1992). However, by quantifying fiber orientation by $\alpha_{\text {helix }}$ alone, the anatomical finding, that myocardial fibers are wrapped between the subendocardial and subepicardial layers (Torrent-Guasp, 1973; Streeter, 1979; Streeter et al., 1978) is ignored. For a complete three-dimensional description of the experimentally observed muscle fiber orientation a second angle is needed, the transverse angle $\alpha_{1 \text { rans }}$ (Fig. 1). The latter angle is defined as the angle between the local circumferential direction and the projection of the fiber path on the plane perpendicular to the local longitudinal direction (Streeter, 1979).

The aim of the present study was to investigate the effect of changes in the spatial distribution of the transverse angle on the left ventricular wall mechanics, using a mathematical model (Bovendeerd, 1990; Bovendeerd et al., 1992). First, a reference simulation (REF) of a complete cardiac cycle was performed, in which the transverse angle was zero and the helix angle was chosen so that active muscle fiber stress was distributed approximately homogeneously across the ventricular wall (Bovendeerd et al., 1992). In a second simulation (TRANS), a small (spatially averaged value $3^{\circ}$ ) nonzero transverse angle was introduced. In a third simulation (HELIX) only the helix angle was 

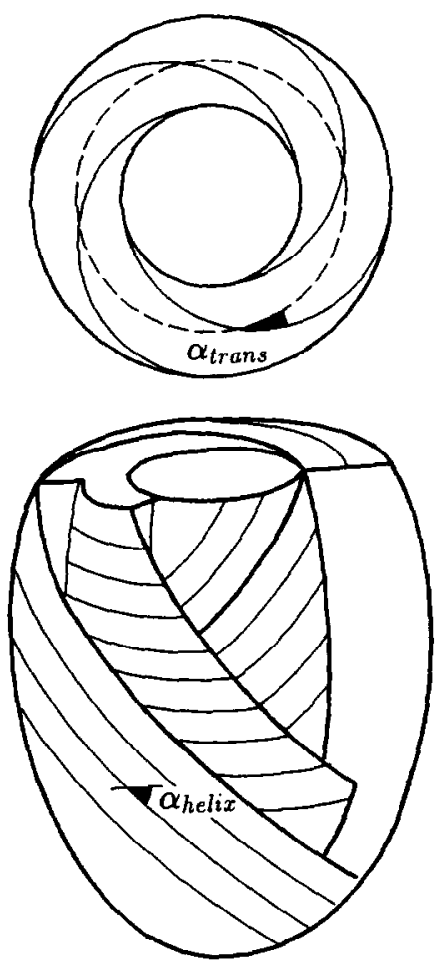

Fig. 1. Quantification of muscle fiber orientation; the bottom panel shows the helix angle $\alpha_{\text {helix }}$, which is negative at the indicated epicardial site; the upper panel shows the transverse angle $\alpha_{\text {trans }}$ in a top view of the basal plane.

changed with respect to the reference simulation. Changes in ventricular wall mechanics in response to changes in transverse and helix angle were compared.

Global pump function and local wall mechanics, as calculated in the three simulations, are presented. Global pump function was quantified by the pressure-volume relation. Local wall mechanics was quantified by fiber strain, rotation of the wall around the base-to-apex axis, and wall stress related to active contraction of the myocardium as well as to deformation of the passive elastic structures.

\section{DESIGN OF THE NUMERICAL MODEL OF LEFT VENTRICULAR MECHANICS}

The design of the numerical model has been described before (Bovendeerd, 1990; Bovendeerd et al. 1992 ) and is recapitulated only briefly.

\section{Geometry and material properties}

In the model the reference state is defined as the situation with zero transmural pressure. Then the endocardial and epicardial surfaces are represented by truncated confocal ellipsoids (Streeter et al., 1973), leaving a thick ventricular wall in between (Fig. 2). The common focal length of the ellipsoids $C$ equals $43 \mathrm{~mm}$. The height $h$ above the equator, at which the ellipsoids are truncated, is taken to be one-third of the

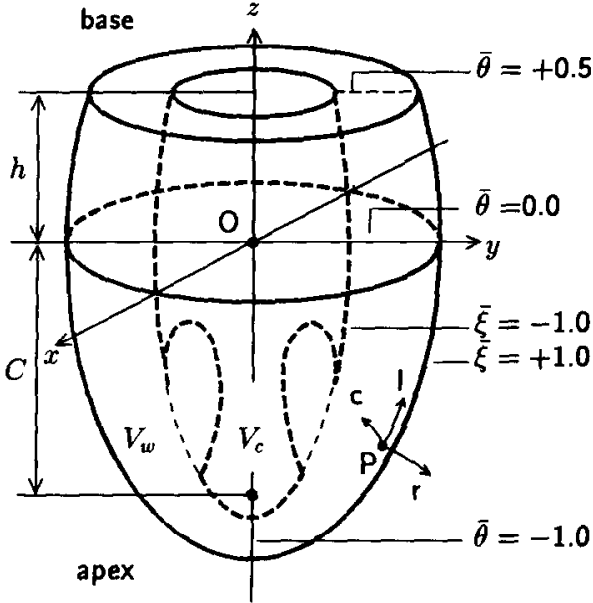

Fig. 2. Geometry of the model of the left ventricle showing the focal length $C(43 \mathrm{~mm})$, the truncation height above the equator $h(24.8 \mathrm{~mm})$, wall volume $V_{\mathrm{w}}(140 \mathrm{ml})$, cavity volume $V_{\mathrm{c}}$ and origin $\mathrm{O}$; in an arbitrary point $\mathrm{P}$ the circumferential (c), longitudinal (1) and radial (r) direction are indicated; coordinate systems $(x, y, z)$ and $(\bar{\theta}, \bar{\xi})$ are explained in the appendix.

midwall base-to-apex length and equals $24.8 \mathrm{~mm}$. The volume of the cavity $\left(V_{\mathrm{c}}\right)$ and the myocardial tissue $\left(V_{w}\right)$, including the papillary muscle volume of $4 \mathrm{ml}$, equals 40 and $140 \mathrm{ml}$, respectively. With the use of these data, the inner and outer equatorial radii and the apical wall thickness can be calculated to be 16.3, 31.3 and $7.2 \mathrm{~mm}$, respectively.

In the model, myocardial tissue is assumed to consist of fluid, connective tissue and muscle fibers. Each constituent contributes to the total Cauchy stress $\sigma$ in the tissue:

$$
\boldsymbol{\sigma}=-p \mathbf{I}+\boldsymbol{\sigma}_{\mathrm{p}}+\sigma_{\mathrm{a}} \mathbf{e}_{\mathbf{i}} \mathbf{e}_{\mathbf{i}} .
$$

The fluid, which is assumed to be trapped in the solid, is subject to a hydrostatic pressure $-p \mathbf{I}, \mathbf{I}$ being the unity tensor. The solid, mainly connective tissue and muscle fibers, can bear a three-dimensional passive stress $\sigma_{\mathrm{p}}$, which is assumed to increase exponentially with strain. The passive constitutive behavior is assumed to be transversely isotropic, the stiffness in the fiber direction $e_{\mathrm{f}}$ being twice as high as that in the cross-fiber direction (Yin et al., 1987). During systole, the degree of anisotropy of the solid increases since an additional active stress component $\sigma_{\mathrm{a}} \mathbf{e}_{\mathrm{r}} \mathbf{e}_{\mathrm{f}}$ is generated in the contractile units in the muscle cells, the sarcomeres. The active stress $\sigma_{\mathrm{a}}$ depends on time, sarcomere length and velocity of sarcomere shortening (de Tombe and ter Keurs, 1990) and is directed along the fiber direction $\mathbf{e}_{\mathrm{f}}$. Generation of active stress is initiated by electrical depolarization of the cell membrane. In the model it is assumed that depolarization of the ventricular wall occurs rotationally symmetrical, starting in the endocardial apical region and reaching the epicardial basal region after about $50 \mathrm{~ms}$. The aortic hemodynamic load is simulated by a 
unidirectional valve, followed by a characteristic resistance in series with an arterial compliance which is in parallel to a peripheral resistance (Westerhof $e t$ al., 1973).

\section{Calculation procedure}

A Galerkin-type finite element method is used to convert the equations of conservation of mass and momentum into a 20-node three-dimensional brick element formulation (Fig. 3). Within the element, the displacement field is approximated by a quadratic interpolation of the displacement of the 20 nodes. Similarly, the hydrostatic pressure field is approximated by a linear interpolation of the pressures in the eight corner nodes.

Initially, the left ventricle is rotationally symmetric with respect to the long axis. No circumferential variation of material properties is assumed. Since the mechanical loading of the ventricle is also axisymmetric, the rotational symmetry of the ventricle is conserved during the complete cardiac cycle. To save computing time, only a quarter section of the ventricle is considered. This quarter section contains 27 elements with a total of 208 nodes (Fig. 3). The displacement and pressure fields in the planes of dissection are coupled using the property of rotational symmetry. Axial displacement of the nodes in the basal plane and circumferential displacement of the nodes in the subendocardial basal ring are suppressed. The endocardial surface is loaded by the cavity pressure. Loading of the epicardium is assumed to be zero during the whole cardiac cycle.

In the simulation of a cardiac cycle typically time steps of $5 \mathrm{~ms}$ are used. At the end of each time in-

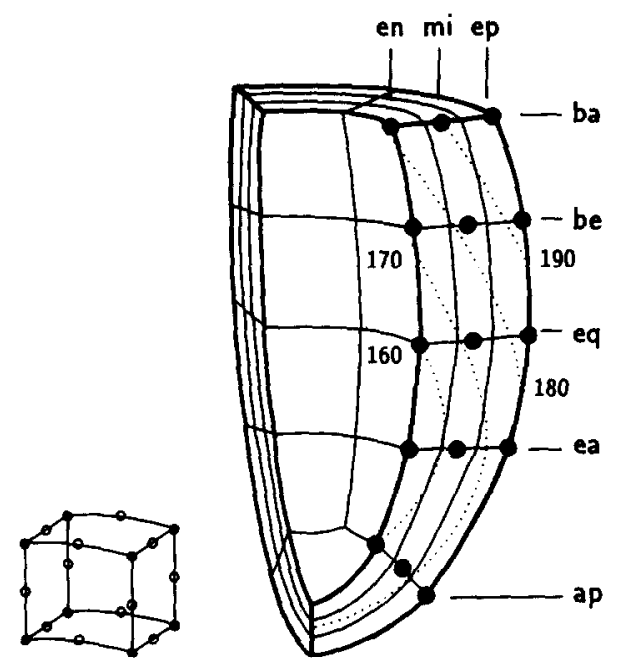

Fig. 3. The 20-node brick element (left) with pressure/displacement nodes $(O)$ and displacement nodes $(O)$ used to compose the finite element mesh of a quarter of the left ventricle (right); moments of activation (in $\mathrm{ms}$ ) are indicated by the dotted lines; local mechanics will be presented at 5 longitudinal levels, base (ba), base-equator (be), equator (eq), equator-apex (ea) and apex (ap) and 3 radial levels, subendocardial (en), midwall (mi) and subepicardial (ep). crement, equilibrium is required between the forces associated with cavity pressure, wall stress and the kinematic boundary conditions. The simulation starts with the diastolic filling phase, during which cavity pressure is prescribed to increase from $0 \mathrm{kPa}$ at $t=0 \mathrm{~ms}$ to $1 \mathrm{kPa}$ at $t=150 \mathrm{~ms}$. At this moment the depolarization wave starts in the apical subendocardial region of the ventricle. The ventricular wall is completely activated after about $50 \mathrm{~ms}$ (Fig. 3). Active stress is generated immediately after depolarization. The diastolic phase ends with the closure of the mitral valve, which is assumed to occur at $t=200 \mathrm{~ms}$. During the following isovolumic contraction phase, cavity pressure is determined iteratively until the cavity volume equals the end-diastolic cavity volume. The ejection phase starts when cavity pressure exceeds the prescribed end-diastolic aortic pressure level of $10 \mathrm{kPa}$. During the ejection phase, cavity pressure is determined iteratively until the decrease of cavity volume matches the aortic volume flow, as determined by the aortic hemodynamic input impedance. The ejection phase ends when the calculated flow reverses. The isovolumic relaxation phase is simulated analogously to the isovolumic contraction phase. The calculation ends at $t=600 \mathrm{~ms}$.

\section{Simulations performed}

Simulations with three different distributions of muscle fiber orientation were performed (Fig. 4). In the REF simulation, the helix angle $\alpha_{\text {helix }}$ varied from $+60^{\circ}$ at the endocardium through $15^{\circ}$ in the midwall layers to $-60^{\circ}$ at the epicardium [Fig. 4(a)], while the transverse angle $\alpha_{\text {trans }}$ was set to zero. In simulation TRANS the distribution of $\alpha_{\text {helix }}$ was identical to that in the REF simulation, but the transverse angle $\alpha_{\text {trans }}$ was assumed to vary transmurally from $0^{\circ}$ at the endocardium through a maximum at the midwall level to $0^{\circ}$ at the epicardium [Fig. 4(b)]. The midwall maximum value of $\alpha_{\text {trans }}$ varied from $+13.5^{\circ}$ at the base through $0^{\circ}$ at the equator to $-13.5^{\circ}$ at the apex [Fig. 4(c)]. In simulation HELIX, $\alpha_{\text {helix }}$ varied linearly from $+60^{\circ}$ at the endocardium, through $0^{\circ}$ in the midwall layers until $-60^{\circ}$ at the epicardium [Fig. 4(a)], while $\alpha_{\text {trans }}$ was kept zero. The choice of the distributions of $\alpha_{\text {helix }}$ and $\alpha_{\text {trans }}$ is elucidated in the discussion and described in more detail in the appendix.

Global pump function, as calculated in the simulations, is characterized by left ventricular pressurevolume loops and changes in geometry. Local deformation is characterized by fiber strain and the angle of rotation of the wall around the ventricular long axis. Local wall stress related to the active contraction of the myocardium is characterized by the active first Piola-Kirchhoff stress $T_{\mathrm{a}}$, defined as:

$$
T_{\mathrm{a}}=\frac{F_{\mathrm{a}}}{A_{\mathrm{o}}}=\sigma_{\mathrm{a}} \frac{l_{\mathrm{s}, 0}}{l_{\mathrm{s}}},
$$

where $F_{\mathrm{a}}$ represents the active force generated by the sarcomere, $A_{0}$ represents the cross-sectional area of 


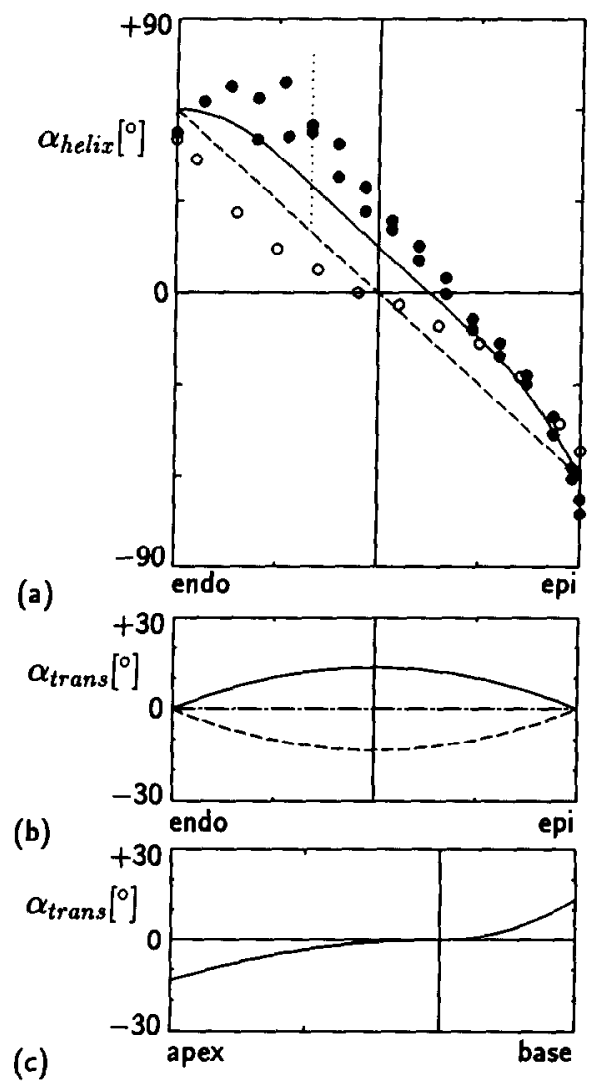

Fig. 4. Muscle fiber orientation: (a) transmural distribution of the helix fiber angle $\alpha_{\text {helix }}$ as assumed in simulations REF and TRANS (-) and in simulation HELIX (- - ) and measured approximately at the equator by Streeter et al. (1969) (O) and Streeter (1979) (O); data from Streeter (1979) were plotted assuming that the distance between the endocardial surface and the trabecula-compacta interface, indicated by the vertical dotted line, equalled one-third of the wall thickness; (b) transmural distribution of the transverse fiber angle $\alpha_{\text {trans }}$ as assumed in simulation TRANS at base $(-)$, equator $(-\cdot-)$ and apex $(---)$; (c) base-to-apex distribution of $\alpha_{\text {trans }}$ in simulation TRANS.

the sarcomere in the reference situation, and $l_{\mathrm{s}}$ and $l_{s, 0}$ represent actual and reference sarcomere length, respectively. As a measure of the magnitude of the stress, related to the deformation of the passive elastic structures in the wall, we use the largest principal Cauchy stress $\sigma_{\mathrm{pp}}$, defined as the passive stress in that direction where the passive stress $\sigma_{p}$ is largest.

\section{RESULTS}

\section{Global hemodynamics and deformation}

In Fig. 5 the left ventricular cavity pressure $p_{\mathrm{c}}$ is plotted as a function of left ventricular cavity volume $V_{c}$ for the simulations REF, HELIX and TRANS. Hemodynamic data are presented in Table 1. In the reference simulation REF, during diastole cavity pressure increased from 0 to $1 \mathrm{kPa}$ causing cavity volume $V_{\mathrm{c}}$ to increase from 40 to $78.3 \mathrm{ml}$. During the isovolumic contraction phase, cavity pressure rose

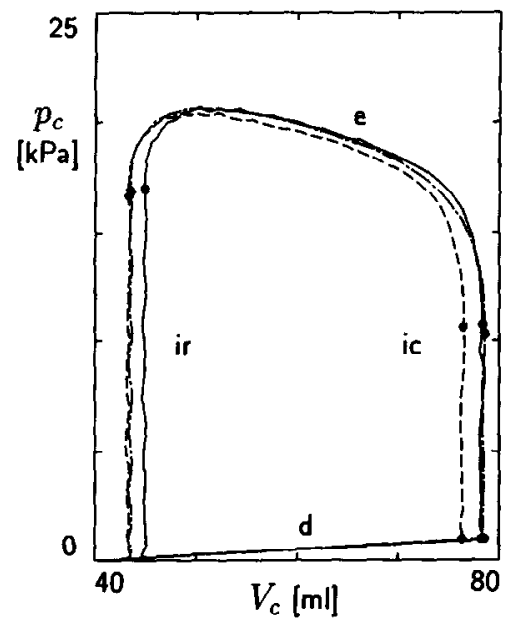

Fig. 5. Left ventricular cavity pressure $p_{\mathrm{c}}$ plotted vs cavity volume $V_{c}$ for simulations REF (-), HELIX $(---)$ and TRANS $(-\cdot)$; dots indicate transitions between the diastolic filling phase (d), the isovolumic contraction phase (ic), the ejection phase (e) and the isovolumic relaxation phase (ir).

until at $t=264 \mathrm{~ms}$ the aortic pressure level $(10 \mathrm{kPa})$ was exceeded causing the aortic valve to open. During ejection, pressure reached a maximum of $20.7 \mathrm{kPa}$, while $33.4 \mathrm{ml}$ of blood was ejected into the aorta. Ejection stopped at $t=442 \mathrm{~ms}$. During the isovolumic relaxation phase, cavity pressure declined until the ventricle became completely passive again. Compared to simulation REF, in simulation TRANS ejection started earlier ( $256 \mathrm{vs} 264 \mathrm{~ms}$ ), lasted longer (190 vs $178 \mathrm{~ms}$ ) and ejected volume was higher (35.1 vs $33.4 \mathrm{ml}$ ). In simulation HELIX global hemodynamics was virtually the same as in simulation REF.

In Fig. 6, the calculated long axis cross-section of the ventricle is shown at five moments during the cardiac cycle. During diastolic filling the wall thinned. During the isovolumic contraction phase, the ventricular diameter increased at the base and decreased near the apex, while the base-to-apex length increased slightly. During ejection, the ventricular diameter remained relatively large at the base. In simulation TRANS, during the ejection phase the basal cavity diameter and the equatorial wall thickness were larger than found in the REF simulation. In the HELIX simulation, the deformation pattern was similar to that in the REF simulation.

\section{Local mechanics}

In Figs 7 and 8, local mechanical quantities as calculated in simulations REF, TRANS and HELIX are shown as a function of time $t$ at the 15 nodes as indicated in Fig. 4. The nodes are located at three radial levels and five longitudinal levels. In describing the results, we first focus on the REF simulation.

At the beginning of the simulation, all sarcomere lengths were set to $2.0 \mu \mathrm{m}$ (Fig. 7, top left panel). During diastole most sarcomeres in the subendocardial and midwall layers were stretched to about 
Table 1. Hemodynamics as calculated in simulations REF, TRANS and HELIX: moment of end-diastole $t_{\text {ed }}$, moment of beginning $t_{\mathrm{be}}$ and end $t_{\mathrm{ec}}$ of the ejection phase, duration of the ejection phase $\Delta t_{\mathrm{e}}$, cavity pressure at beginning $p_{\mathrm{bc}}$ and end $p_{e e}$ of the ejection phase, maximum cavity pressure $p_{\max }$, cavity volume at beginning $V_{b e}$ and end $V_{e c}$ of the ejection phase, and ejected volume $\Delta V_{\mathrm{e}}$

\begin{tabular}{lcccccccccc}
\hline Simulation & $\begin{array}{c}t_{\mathrm{ed}} \\
(\mathrm{ms})\end{array}$ & $\begin{array}{c}t_{\mathrm{bc}} \\
(\mathrm{ms})\end{array}$ & $\begin{array}{c}t_{\mathrm{ec}} \\
(\mathrm{ms})\end{array}$ & $\begin{array}{c}\Delta t_{\mathrm{e}} \\
(\mathrm{ms})\end{array}$ & $\begin{array}{c}p_{\mathrm{be}} \\
(\mathrm{kPa})\end{array}$ & $\begin{array}{c}p_{\mathrm{ec}} \\
(\mathrm{kPa})\end{array}$ & $\begin{array}{c}p_{\max } \\
(\mathrm{kPa})\end{array}$ & $\begin{array}{c}V_{\mathrm{be}} \\
(\mathrm{ml})\end{array}$ & $\begin{array}{c}V_{\mathrm{ec}} \\
(\mathrm{ml})\end{array}$ & $\begin{array}{c}\Delta V_{\mathrm{c}} \\
(\mathrm{ml})\end{array}$ \\
\hline REF & 200 & 264 & 442 & 178 & 10.7 & 20.7 & 17.0 & 78.3 & 44.9 & 33.4 \\
TRANS & 200 & 256 & 446 & 190 & 10.3 & 20.6 & 16.9 & 78.6 & 43.5 & 35.1 \\
HELIX & 200 & 262 & 442 & 180 & 10.6 & 20.4 & 16.7 & 76.4 & 43.3 & 33.1 \\
\hline
\end{tabular}

ref
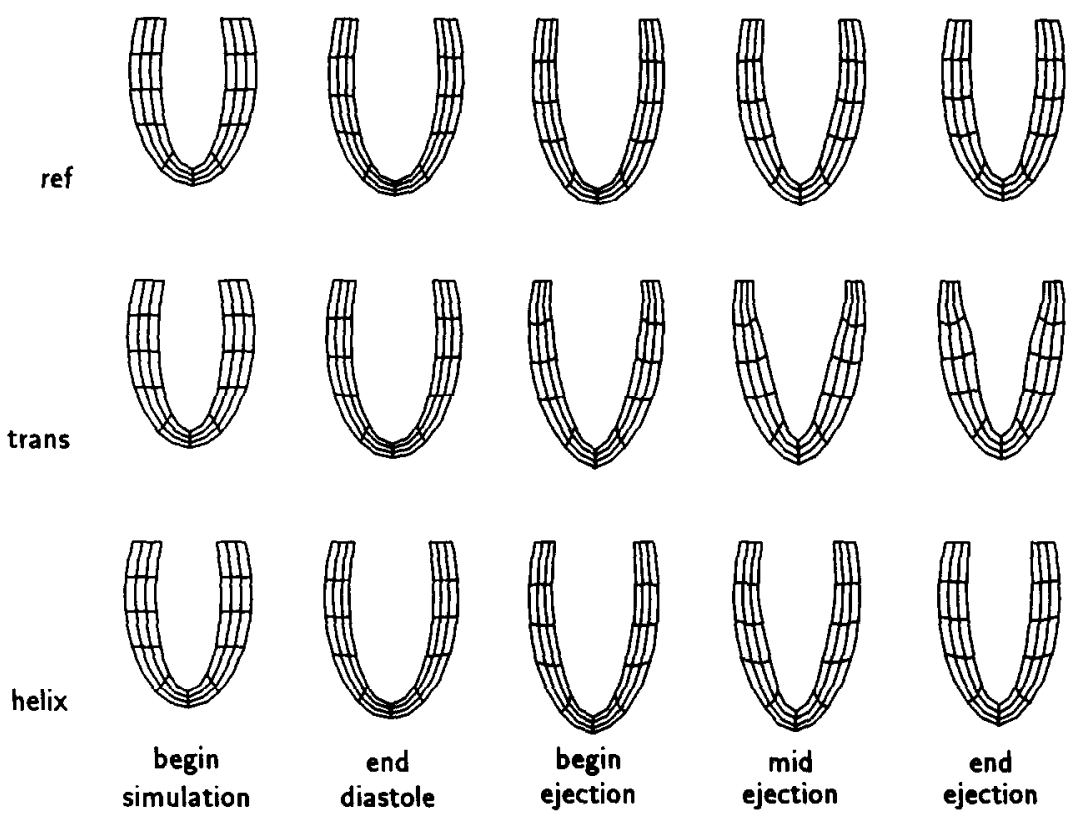

Fig. 6. Shape of a ventricular long axis cross-section at 5 moments during the cardiac cycle as calculated in simulations REF, TRANS and HELIX.

$2.20-2.25 \mu \mathrm{m}$. In the subepicardial layer sarcomere stretch was slightly less. In the early activated region $(t<170 \mathrm{~ms}$, Fig. 3) near the apical subendocardium sarcomeres shortened quickly during the isovolumic contraction phase, the velocity of sarcomere shortening decreased at the beginning of the ejection phase, and sarcomeres already lengthened at the end of the ejection phase. Compared to this pattern, in later activated regions $(170 \mathrm{~ms}<t<185 \mathrm{~ms})$, sarcomere shortening during isovolumic contraction was less, and velocity of sarcomere shortening did not change at the beginning of the ejection phase. The rate of sarcomere shortening became zero at the end of the ejection phase. In the last activated basal region ( $t>185 \mathrm{~ms}$ ) sarcomeres lengthened slightly during the isovolumic contraction phase. During ejection, the rate of sarcomere shortening was relatively high, and shortening continued during the first part of the isovolumic relaxation phase. After complete relaxation all sarcomeres were slightly longer than $2.0 \mu \mathrm{m}$, while cavity volume was slightly larger than at the beginning of the simulation.
In the bottom left panel of Fig. 7 the angle of rotation of the 15 nodes in the ventricular wall around the long axis is shown as a function of time $t$. At the base, endocardial rotation was prohibited to prevent rigid body rotation of the whole ventricle. In the remaining points, during the diastolic filling phase rotation was slightly positive, i.e. the cross-section rotated counterclockwise observing the ventricle from the base. During the isovolumic contraction phase the angle of rotation increased strongly. In the basal part rotation was considerably more in the subepicardial than in the subendocardial layers. In the apical half this pattern was reversed. During the ejection phase, rotation of the basal epicardial layers was positive while in the apical region rotation was negative. At the end of the ejection phase transmural differences in the angle of rotation at the base, the equator and the apex were approximately $+35,0$ and $-35^{\circ}$, respectively. During the isovolumic relaxation phase the ventricle rotated back to the diastolic state.

In the top left panel of Fig. 8 the change of the active first Piola-Kirchhoff muscle fiber stress is 

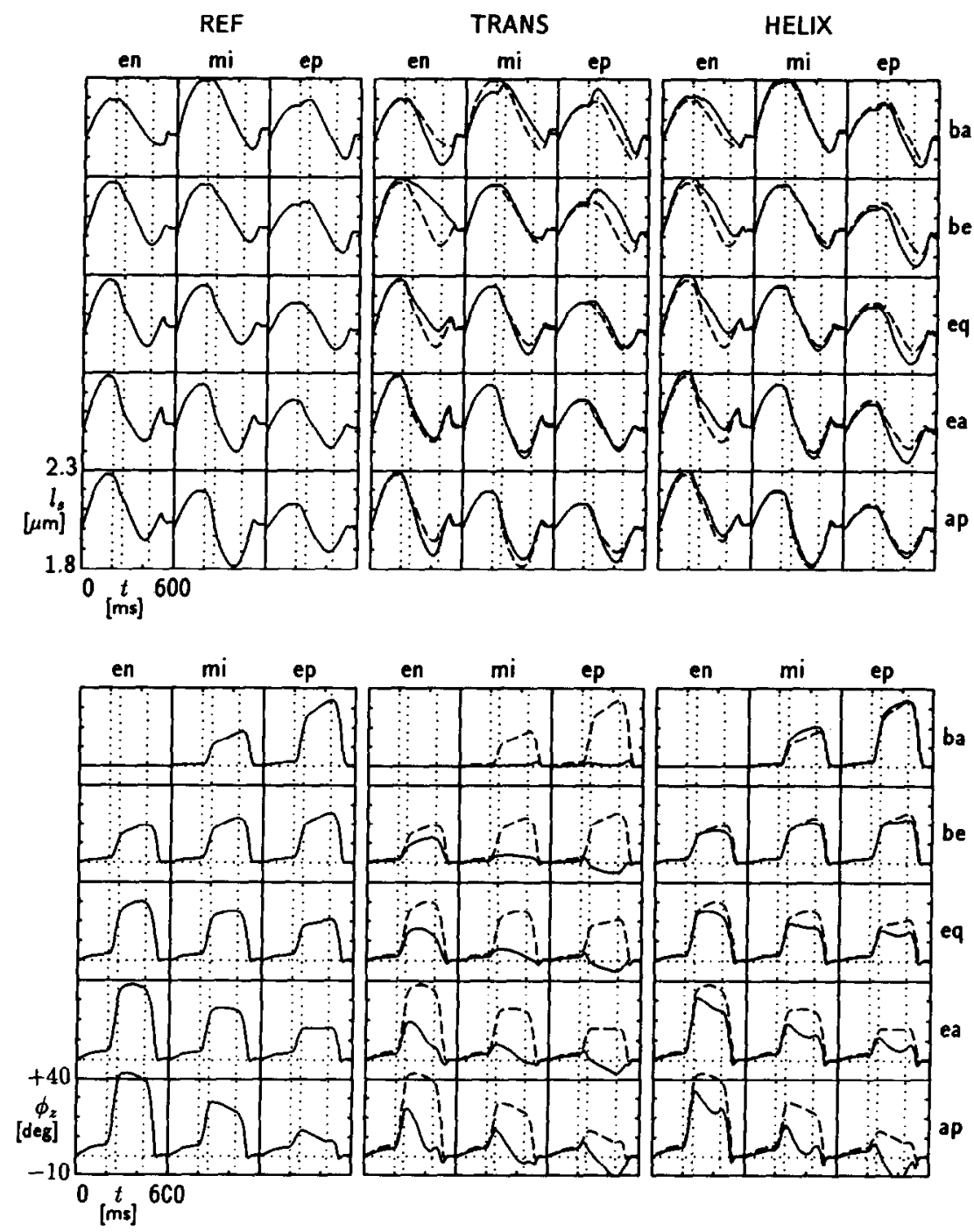

Fig. 7. Local wall deformation as calculated in simulations REF (left panel), TRANS (middle panel) and HELIX (right panel); sarcomere length $l_{s}$ (top) and angle of rotation around the LV long axis $\phi_{2}$ (bottom) are plotted as a function of time $t$ for the 15 nodes indicated in Fig. 3; the dashed lines in the middle and right panels indicate tracings for the REF simulation; the vertical dotted lines indicate the end of the diastolic phase and the beginning and end of the ejection phase.

shown as a function of time $t$. During the isovolumic contraction phase active stresses increased rapidly. During the ejection phase active stresses were distributed rather homogeneously across the major part of the wall, reaching a maximum of about $50 \mathrm{kPa}$. Near the apex active stresses were slightly lower and distributed less homogeneously.

In the bottom left panel of Fig. 8, the largest principal Cauchy stress in the passive tissue is plotted as a function of time. At the end of diastole, passive stresses were less than $5 \mathrm{kPa}$. During the ejection phase, in the major part of the wall the magnitude of the passive stresses was of the order of $10 \%$ of the corresponding active muscle fiber stresses. At the basal and the apical level, however, the magnitude of the passive stresses approached the level of the active muscle fiber stress.
Local mechanics as calculated in simulation TRANS is shown in the middle panels of Figs 7 and 8. Comparison of the results for the TRANS (solid tracings) and the REF (dashed tracings) simulation reveals the effect of introducing a radial component in the muscle fiber path. Generally, diastolic sarcomere stretch was unaffected (Fig. 7). Compared to the RFF simulation, in the equatorial region during the ejection phase sarcomeres tended to be longer in the outer layers and shorter in the midwall layer. Consequently, active muscle fiber stress was higher in the outer layers than in the midwall layer (Fig. 8). In the basal and apical region, a similar correspondence between systolic sarcomere length and active fiber stress was found. The largest effect of the introduction of a nonzero transverse fiber angle was found in the pattern of rotation of the wall around the long axis (Fig. 7). Across the 

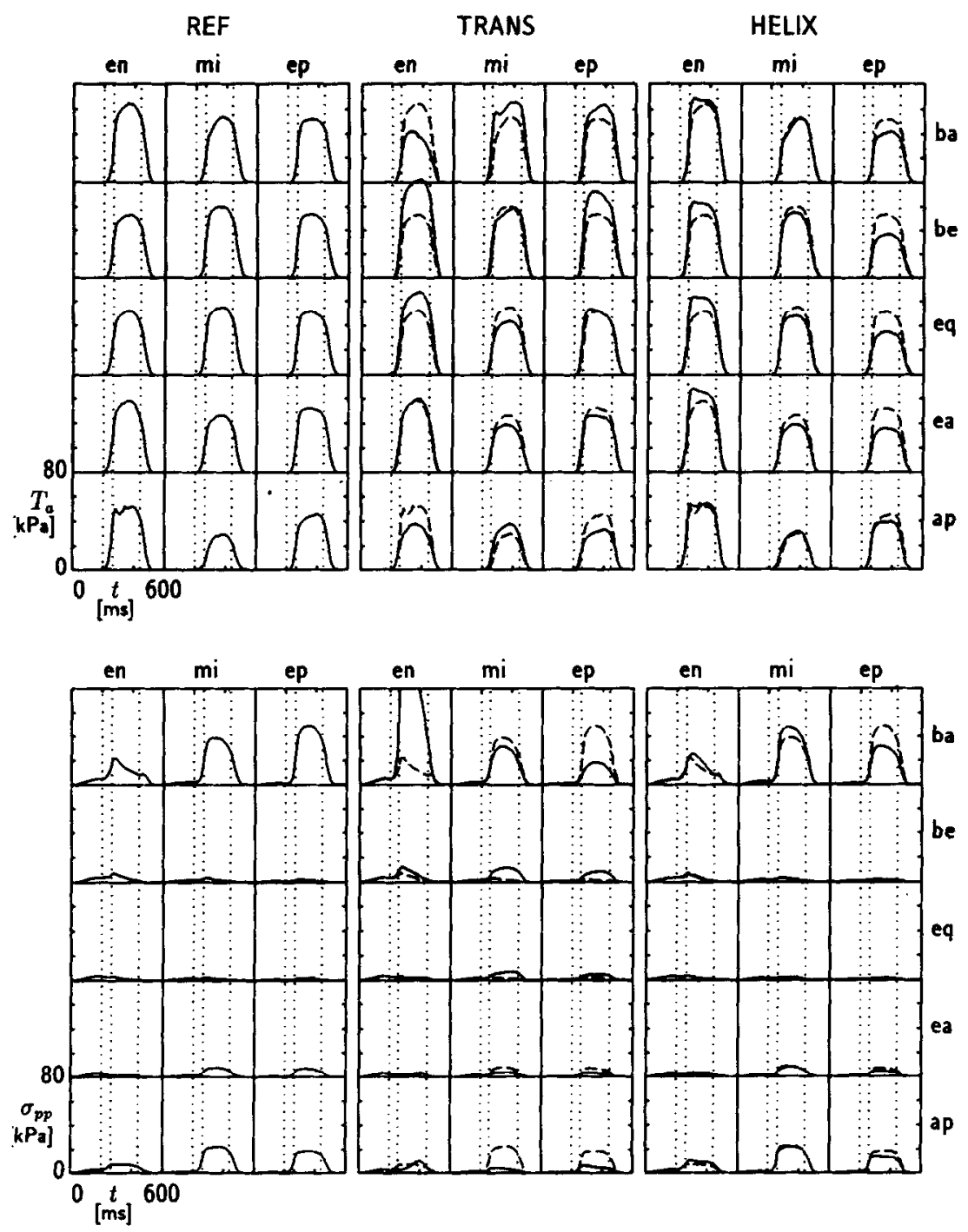

Fig. 8. Local wall stress as calculated in simulations REF, TRANS and HELIX; active first Piola-Kirchhoff fiber stress $T_{\mathrm{a}}$ (top) and largest principal passive Cauchy stress $\sigma_{\mathrm{pp}}$ (bottom) are plotted as a function of time $t$ according to the format of Fig. 7 .

entire wall, both the magnitude and the transmural differences of the angle of rotation decreased considerably. At the basal level virtually no rotation was found during the entire cardiac cycle. At the remaining levels the angle of rotation decreased from subendocardium to subepicardium, leaving transmural differences of about $15^{\circ}$. At the basal level, maximum passive stresses increased and shifted from the subepicardium to the subendocardium (Fig. 8). At the apical level, passive stresses were significantly smaller than in the REF simulation.

Local mechanics as calculated in the HELIX simulation is shown in the right panels of Figs 7 and 8 . Comparison of the results for the HELIX simulation (solid lines) with those of the REF simulation (dashed lines) reveals the influence of a change of the transmural distribution of the helix fiber angle. In Fig. 7, it can be observed that during the diastolic filling phase, in general sarcomere stretch was increased in the subendocardial region and decreased in the subepicardial region. Near the equator, the endocardial-epicardial difference in sarcomere length was maintained during the isovolumic contraction and the ejection phase. The transmural gradient of sarcomere length is reflected in a similar gradient of active muscle fiber stress from about $60-70 \mathrm{kPa}$ in the subendocardial layers to about $30-40 \mathrm{kPa}$ in the subepicardial ones (Fig. 8). As compared to the REF simulation, systolic angles of rotation were less, especially in the apical region (Fig. 7). The transmural differences in rotation remained virtually unchanged. The distribution of the passive stresses was quite similar in the HELIX and the REF simulation (Fig. 8). 


\section{DISCUSSION}

\section{Setup of the model}

In the model many aspects of the ventricular anatomy and physiology are incorporated: the thickwalled geometry and the varying muscle fiber orientation across the wall, the anisotropic, nonlinear stress-strain behavior of the passive myocardial tissue, the dependence of active stress on time, strain and strain rate, the activation sequence of the ventricular wall and the aortic afterload.

The setup of the model is three-dimensional, enabling simulation of the left ventricular mechanics without assuming any symmetry conditions (Bovendeerd, 1990; Bovendeerd et al., 1991). In this study we assumed axisymmetry to facilitate the definition of fiber orientation and the analysis of the effects of changes in this orientation.

The transversly isotropic description of the passive material behavior seems reasonable in view of the structure of this tissue (Caulfield and Borg, 1979) and the results of biaxial loading experiments (Yin et al., 1987). In the latter experiments, a mean degree of anisotropy of 2 was found, with a maximum of 3 . Setting the degree of anisotropy to 3 instead of 2 in the present simulations is expected to have but a minor influence of the results, since the increased passive stress in the fiber direction will still be small with respect to the active fiber stress. A larger influence might be expected from changes in shear behavior of the passive tissue. This aspect will be discussed later in this section.

In the model we neglected viscous properties of the passive tissue (Huyghe et al., 1991a), motion of the intracoronary blood with respect to the solid (Huyghe et al., 1991b), and residual stresses and strains (Omens and Fung, 1990). This simplification seems to be allowed because the stresses, associated with these properties, are typically in the range of $1 \mathrm{kPa}$, which is small as compared to the active fiber stresses during systole (order $50 \mathrm{kPa}$ ), which phase is the focus of the present study.

In the model, muscle fiber orientation is quantified by the helix and transverse fiber angle (Fig. 1). In the experimental assessment of the spatial distribution of $\alpha_{\text {helix }}$ and $\alpha_{\text {trans, }}$, difficulties are encountered related to (1) the definition of the circumferential, longitudinal and radial directions in the real ventricular wall, (2) the definition of the endocardial surface, because of the presence of the trabecular layer, (3) the branching of the muscle fibers, and (4) the artifacts introduced by the postmortem measuring procedure. Reported values of $\alpha_{\text {helix }}$ range from -80 to $-50^{\circ}$ in the subepicardial layers and from +20 to $+80^{\circ}$ in the subendocardial layers (Greenbaum et al, 1981; Ross and Streeter, 1975; Streeter, 1979; Streeter et al., 1969). The transverse angle $\alpha_{\text {irans }}$ is positive in the basal half and negative in the apical half of the ventricle (Anderson and Becker, 1980; Torrent-Guasp, 1973). Quantitative information on the spatial distribution of $\alpha_{1 \text { rans }}$ is limited. Below the equator, measured values of $\alpha_{\text {trans }}$ range from -30 to $0^{\circ}$, with an average value of $-8.4 \pm 1.0^{\circ}$ (Streeter et al., 1978). In another study (Streeter, 1979) the value of $\alpha_{\text {trans }}$, averaged across the wall thickness, was found to be $-4.6 \pm 0.8^{\circ}$ near the apex and $-3.5 \pm 0.6^{\circ}$ halfway between equator and apex. In the latter study, a simplified model was proposed, in which $\alpha_{\text {trans }}$ equalled $+3^{\circ}$ in the entire basal half of the $\mathrm{LV}$ and $-3^{\circ}$ in the entire apical half.

In our model, in the reference simulation the transmural distribution of the helix angle was chosen so [Fig. 4(a)] that during the ejection phase active muscle fiber stress was distributed approximately homogeneously across the ventricular wall (Bovendeerd et al., 1992). The linear transmural variation of $\alpha_{\text {helix }}$, as assumed in simulation HELIX, was chosen because it is often used in models describing the left ventricular mechanics. Both choices for $\alpha_{\text {helix }}$ are within the range of reported physiological values (Streeter, 1979; Streeter et al., 1969) [Fig. 4(a)]. In the TRANS simulation, the transmural variation of $\alpha_{\text {trans }}$ was assumed to be quadratic, with a maximum at midwall [Fig. 4(b)]. $\alpha_{\text {trans }}$ was set to zero at the endocardial and epicardial surfaces since muscle fibers are not supposed to end at these surfaces. In the longitudinal direction, $\alpha_{\text {trans }}$ was assumed to vary quadratically with the distance from the equatorial plane, $\alpha_{\text {trans }}$ being positive above and negative below the equator [Fig. 4(c)]. The magnitude of $\alpha_{\text {trans }}$ was choosen so that the spatially averaged value of $\alpha_{\text {trans }}$ equals $+3^{\circ}$ in the basal half of the $\mathrm{LV}$ and $-3^{\circ}$ in the apical half (Streeter, 1979).

\section{RESULTS}

The change of the helix fiber angle in simulation HELIX resulted in a stiffer passive ventricle, as compared to the reference situation, whereas ejected volume was unchanged (Fig. 5). The introduction of a nonzero transverse angle in simulation TRANS, yielded an increase of ejected volume of about $5 \%$, although the contractile properties of the sarcomeres were identical in the REF and TRANS simulation.

The change in the helix angle affected the global deformation pattern of the ventricle only slightly. As compared to simulations REF and HELIX, in simulation TRANS basal wall thickness was small and basal ventricular diameter was large. The pronounced basal circumferential lengthening is related to the fact that the stiffest elements in the myocardial tissue, the muscle fibers, are no longer fully oriented in the circumferential direction, causing a more compliant behavior along the circumferential direction.

Within the wall, the applied variations of the helix and transverse fiber angle modified the distributions of sarcomere length and active muscle fiber stress to a similar amount (top panels of Figs 7 and 8). With respect to the REF simulation, sarcomere length changed up to $0.1 \mu \mathrm{m}$, while systolic active fiber stress changed up to $50 \%$. In both simulations the distribu- 
tion of active muscle fiber stress across the $L V$ wall was less uniform than in the REF simulation.

Transmural differences in the angle of rotation are a response to the transmural shear load that results from the active stresses in the muscle fibers, acting along a direction that varies transmurally. In simulations REF and HELIX, this shear load can only be counteracted by the passive myocardial tissue. In simulation TRANS, however, the mechanical coupling between the subendocardial and the subepicardial layers is also born by the myocardial fibers. Since these fibers are much stiffer than the passive tissue, transmural shear deformation in simulation TRANS is strongly reduced: in comparison to simulation REF, in simulation TRANS at the base the transmural difference in angle of rotation was reduced from $35^{\circ}$ to virtually zero, while at the apex a reduction from 35 to $15^{\circ}$ was found (Fig. 7).

Of course, the transmural shear deformation of the wall depends not only on the transmural shear load of the myocardial tissue but also on the ability of the tissue to resist this load. Since, to the best of our knowledge, no experimental data on the mechanical behavior of passive cardiac tissue under shear stress have been published, the shear stiffness of the tissue, as assumed in our model is quite arbitrary. Thus, we cannot draw conclusions from the magnitude of the radial-circumferential shear deformation as found in the simulations. However, since in simulations REF and TRANS the constitutive behavior of the passive tissue was identical, we may conclude that the reduced shear deformation in simulation TRANS reflects a reduced shear loading of the passive tissue. Nevertheless, we found an increase of the largest principal passive Cauchy stress at the basal endocardium (Fig. 8). This increase is directly related to the increase of the basal diameter, as discussed above. Since the latter diameter seems to be rather high as compared to the physiological situation, the present model should be improved by considering more carefully the physiological basal boundary conditions, associated with the presence of the annuli fibrosi.

\section{Comparison with experimental results}

Since measurement of ventricular wall stress is practically impossible (Huisman et al., 1980), the results of the simulations are evaluated by the comparison of calculated and measured deformation. We focus on circumferential-radial shear strain, since the calculations show that introduction of a nonzero transverse fiber angle primarily affects this strain component. In Fig. 9, circumferential-radial Green-Lagrange shear strains, $E_{\mathrm{cr}}$ as calculated in simulations REF and TRANS, are plotted versus normalized cavity volume $V_{\mathrm{c}} / V_{\mathrm{w}}$. During the diastolic filling and the ejection a virtual linear relation between strain and volume is found. Generally, isovolumic change of strain is larger in the REF simulation than in the TRANS simulation. The change of strain during the

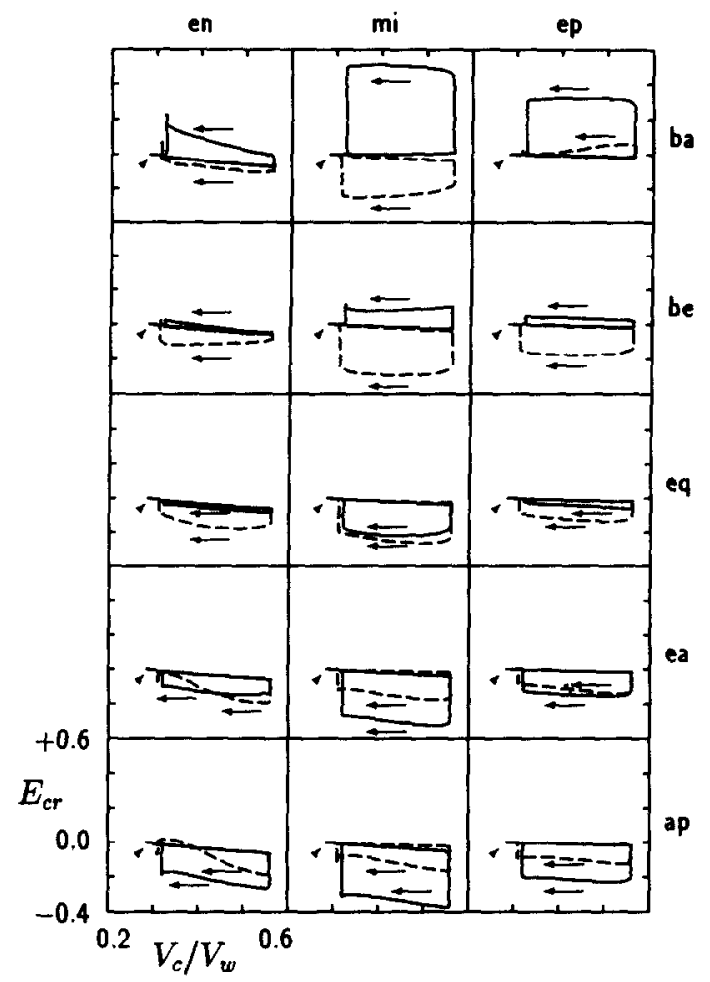

Fig. 9. Circumferential-radial Green-Lagrange strain component $E_{\mathrm{cr}}$ plotted vs normalized cavity volume $V_{\mathrm{c}} / V_{\mathrm{w}}$ at the 15 nodes in the ventricular wall, as indicated in Fig. 3 for simulation REF ( - ) and TRANS (- - ); starting point of the tracings is indicated by the arrowhead, while the ejection phase is indicated by the arrow.

isovolumic phases depends strongly on the position in the ventricular wall.

Douglas et al. (1991) followed radiopaque markers inserted in the ventricular wall using biplane radiography. From the mutual displacements of the markers three-dimensional strains were calculated, referred to the end-diastolic state at maximum volume. For comparison we focus at node 'eq-mi', which corresponds approximately to their site of measurement. The virtually linear relationship between strain and cavity volume, as found in the simulations, was also observed in the experiment. In our simulations the slope of the strain-volume relation was negative both during filling and ejection. In the experiment, this slope was positive during filling, but negative during ejection [one heart, see $E_{13}$ in Fig. 4 of Douglas et al., (1991)]. In the experiment the strain-volume lines during filling and ejection intersected at a cavity volume within the physiological range, and, consequently, circumferential-radial shear strain could either decrease or increase during isovolumic contraction and relaxation, depending on the actual cavity volume. In our simulations, $E_{\mathrm{cr}}$ decreased during isovolumic contraction and increased during relaxation. A more quantitative comparison with the experimental data is difficult because of different choices of the reference state. 
Using the same technique as Douglas et al. (1991), Waldman et al. (1985) determined changes of strain as a function of time in one heart at a location corresponding approximately to our location 'en-ea' and 'mi-ea'. Strains were referred to the end-diastolic state. Circumferential-radial shear strain increased during isovolumic contraction and decreased during ejection [see $E_{13}$ in Fig. 4 of Waldman et al. (1985)], which is opposite to the finding in our study. A more detailed comparison with the experimental results is hampered by the different choices of the reference state and lack of information on cavity volume.

In the study of Buchalter et al. (1990), changes in the angle of rotation around the long ventricular axis with respect to end-diastole were measured using magnetic resonance imaging. Transmural differences in angle of rotation at the end of the ejection phase were reported to vary monotonously from $0^{\circ}$ at the base to about $8^{\circ}$ at the apex. Thus, the results obtained in the TRANS simulation seem to be more realistic than those obtained in the REF and HELIX simulation. However, it should be noted that the agreement of this combined effect of isovolumic contraction and ejection, does not mean that the rotational behavior during each phase separately is predicted realistically. A more quantitative comparison is also hampered by missing information on the volume change.

We conclude that detailed comparison of circumferential-radial shear strains as calculated in this model study with experimentally determined strains is difficult because of different choices of the reference state, missing information on cavity volume, or presentation of the combined effect of more than one phase in the cardiac cycle. Nevertheless, qualitative discrepancies are observed between calculated and measured strains. To enable a quantitative comparison, we suggest measurement of the relation between strain and cavity volume during diastolic filling and ejection separately. Cavity volume should be normalized with respect to wall volume, and strains should be referred to a well-defined state. To make this reference state relatively independent of the passive material properties, the state of zero cavity pressure, or the state where cavity volume equals $30 \%$ of the wall volume would be suitable. Finally, the measurements should be performed at sites where the effect of the transverse angle is largest, i.e. in the basal and apical region.

\section{CONCLUSION}

The simulations showed that the spatial distributions of local ventricular wall stress and strain were very sensitive to the spatial distribution of muscle fiber orientation, modelled in terms of the helix and the transverse fiber angle. The distribution of the transmural differences in angle of rotation around the ventricular long axis was affected predominantly by changes in the spatial distribution of the transverse fiber angle. Moreover, introduction of the transmural crossover of the muscle fibers seemed to reduce the transmural shear loading of the passive tissue.

A comparison of circumferential-radial shear strains, as calculated in this model study, with experimentally determined strains revealed qualitative discrepancies. This is not surprising in view of the large sensitivity of the circumferential-radial shear strains to changes in the transverse fiber angle on the one hand, and the rather arbitrary choice of the transverse fiber angle in the model because of the very limited amount of experimental data on the other.

\section{REFERENCES}

Anderson, R. H. and Becker, A. E. (1980) Cardiac Anatomy. Gower Medical Publishing, London.

Arts, T., Veenstra, P. C. and Reneman, R. S. (1982) Epicardial deformation and left ventricular wall mechanics during ejection in the dog. Am. J. Physiol. 243, H379-H390.

Beyar, R. and Sideman, S. (1984) A computer study of the left ventricular performance based on fiber structure, sarcomere dynamics, and transmural electrical propagation velocity. Circ. Res. 55, 258-375.

Bovendeerd, P. H. M. (1990) The mechanics of the normal and ischemic left ventricle during the cardiac cycle: a numerical and experimental analysis. Ph.D. thesis, University of Limburg, Maastricht, The Netherlands.

Bovendeerd, P. H. M., Arts, T., Huyghe, J. M., van Campen, D. H. and Reneman, R. S. (1992) Dependence of local left ventricular wall mechanics on myocardial fiber orientation: a model study. J. Biomechanics 25, 1129-1140.

Bovendeerd, P. H. M., Arts, T., Huyghe, J. M., van Campen, D. H. and Reneman, R. S. (1991) The mechanics of the ischemic left ventricle during the cardiac cycle. In 1991 Advances in Bioengineering, BED-Vol. 20 (Edited by Vanderby Jr., R.), pp. 651-654. Am. Soc. Mech. Eng., New York.

Buchalter, M. B., Weiss, J. L., Rogers, W. J., Zerhouni, E. A., Weisfeldt, M. L., Beyar, R. and Shapiro, E. P. (1990) Noninvasive quantification of left ventricular rotational deformation in normal humans using magnetic resonance imaging myocardial tagging. Circ. 81, 1236-1244.

Caulfield, J. B. and Borg, T. K. (1979) The collagen network of the heart. Lab. Invest. 40, 364-372.

Douglas, A. S., Rodriguez, E. K., O'Dell, W. and Hunter, W. C. (1991) Unique strain history during ejection in canine left ventricle. Am. J. Physiol. 260, H1591-H1611.

Greenbaum, R. A., Ho, S. Y., Gibson, D. G., Becker, A. E. and Anderson, R. H. (1981) Left ventricular fibre architecture in man. Br. Heart J. 45, 248-263.

Horowitz, A., Perl, M., Sideman, S. and Ritman, E. (1986) Comprehensive model for the simulation of left ventricle mechanics: Part 2. Implementation and results analysis. Med. Biol. Engng Comput. 24, 150-156.

Huisman, R. M., Elzinga, G. and Westerhof, N. (1980) Measurement of left ventricular wall stress. Cardiovasc. Res. 14, 142-153.

Huyghe, J. M., van Campen, D. H., Arts, T. and Heethaar, R. M. (1991a) The constitutive behaviour of passive heart muscle tissue: a quasi-linear viscoelastic formulation. $J$. Biomechanics 24, 841-849.

Huyghe, J. M., van Campen, D. M., Arts, T. and Heethaar, R. M. (1991b) A two-phase finite element model of the diastolic left ventricle. J. Biomechanics 24, 527-538.

Huyghe, J. M., Arts, T., van Campen, D. H. and Reneman, R. S. (1992) Porous medium finite element model of the beating left ventricle. Am. J. Physiol. 262, H1256-H1267. 
Omens, J. H. and Fung, Y. C. (1990) Residual strain in rat left ventricle. Circ. Res. 66, 37-45.

Prinzen, T. T., Arts, T., Prinzen, F. W. and Reneman, R. S. (1986) Mapping of epicardial deformation using a video processing technique. J. Biomechanics 19, 263-273.

Ross, M. A. and Streeter Jr., D. D. (1975) Nonuniform subendocardial fiber orientation in the normal macaque left ventricle. Eur. J. Cardiol. 3, 229-247.

Streeter Jr., D. D. (1979) Gross morphology and fiber geometry of the heart. In Handbook of Physiology-The Cardiovascular System I, Vol. 1. The Heart (Edited by Berne, R. M.), Ch. 4, pp. 61-112. Am. Physiol. Soc., Bethesda, MD.

Streeter Jr., D. D. and Hanna, W. T. (1973) Engineering mechanics for successive states in canine left ventricular myocardium. Circ. Res. 33, 639-655.

Streeter Jr., D. D., Powers, W. E., Ross, M. A. and TorrentGuasp, F. (1978) Three-dimensional fiber orientation in mammalian left ventricular wall. In Cardiovascular System Dynamics (Edited by Baan, J., Noordergraaf, A and J. Raines), pp. 73-84. MIT Press, Cambridge, MA.

Streeter Jr., D. D., Spotnitz, H. M., Patel, D. P., Ross Jr., J. R. and Sonnenblick, E. H. (1969) Fiber orientation in the canine left ventricle during diastole and systole. Circ. Res. 24, 339-347.

\section{APPENDIX}

The geometry of the left ventricle has been described in ellipsoid coordinates $(\xi, \theta, \phi)$, denoting the radial, longitudinal and circumferential direction, respectively (Fig. 2). The ellipsoid coordinates are related to Cartesian coordinates $(x, y, z)$ according to:

$$
\begin{aligned}
& x=C \sinh (\xi) \sin (\theta) \cos (\phi), \\
& y=C \sinh (\xi) \sin (\theta) \sin (\phi), \\
& z=C \cosh (\xi) \cos (\theta),
\end{aligned}
$$

where $C$ represents the common focal length of the ellipsoids and $0 \leqslant \theta \leqslant \pi, 0 \leqslant \phi \leqslant 2 \pi$. Surfaces of constant $\xi$ and constant $\theta$ correspond to confocal ellipsoids and hyperboloids, respectively. For calculation purposes, local normalized coordinates $(\bar{\xi}, \bar{\theta})$ are defined in a plane of constant $\phi$. In contrast to the ellipsoid coordinates $\xi$, the normalized coordinate $\bar{\xi}$ varies linearly with the actual distances in the ventricular wall, from $\bar{\xi}=-1$ at the endocardial surface to $\bar{\xi}=+1$ at the epicardial surface. The normalized longitudinal coordinate $\bar{\theta}$ varies linearly with the distance from the equatorial plane, as measured along the meridional curve through the point of interest. It varies from +0.5 in the basal plane, through 0 at the equator to -1 at the apex.

In the model, the spatial distribution of $\alpha_{\text {helix }}$ and $\alpha_{\text {trans }}$ is a function of these local coordinates $(\bar{\xi}, \bar{\theta})$ :

$$
\alpha_{h e l i x}(\bar{\xi})=\alpha_{h 0}+\alpha_{h 1} \bar{\xi}+\left\{\begin{array}{cc}
4 \alpha_{h 2, \text { en }}(\bar{\xi}+0.5)^{2}, & -1.0 \leqslant \bar{\xi}<-0.5, \\
0, & -0.5 \leqslant \bar{\xi} \leqslant+0.5, \\
4 \alpha_{h 2, e p}(\bar{\xi}-0.5)^{2}, & +0.5<\bar{\xi} \leqslant+1.0,
\end{array}\right.
$$

$$
\alpha_{\text {trans }}(\bar{\xi}, \bar{\theta})=\left\{\begin{array}{cc}
4 \alpha_{12}\left(1-\bar{\xi}^{2}\right) \bar{\theta}^{2} & \bar{\theta}>0, \\
-\alpha_{12}\left(1-\bar{\xi}^{2}\right) \bar{\theta}^{2} & \bar{\theta} \leqslant 0 .
\end{array}\right.
$$

de Tombe, P. P. and ter Keurs, H. E. D. J. (1990) Force and velocity of sarcomere shortening in trabeculae from rat heart: effects of temperature. Circ. Res. 66, 1239-1254.

Torrent-Guasp, F. (1973) The Cardiac Muscle. Fundacin Juan, Madrid.

Waldman, L. K., Fung, Y. C. and Covell, J. W. (1985) Transmural myocardial deformation in the canine left ventricle: normal in vivo three-dimensional finite strains. Circ. Res. 57, 152-163.

Westerhof, N., Elzinga, G. and van den Bos, G. C. (1973) Influence of central and peripherical changes on the hydraulic input impedance of the systemic arterial tree. $\mathrm{Med}$. biol. Engng 11, 710-723.

Yin, F. C. P., Strumpf, R. K., Chew, P. H. and Zeger, S. L. (1987) Quantification of the mechanical properties of noncontracting canine myocardium under simultaneous braxial loading. J. Biomechanics 20, 577-589.
Table 2 contains the parameter values as used in the three simulations. In Fig. 4 the angles $\alpha_{\text {helix }}(\bar{\xi})$ and $\alpha_{\text {inass }}(\bar{\xi}, \bar{\theta})$ were shown graphically.

Table 2. Values of the parameters describing the spatial distributions of the helix fiber angle $\alpha_{\text {helix }}$ and the transverse fiber angle $\alpha_{\text {irnss }}$

\begin{tabular}{lrrrrr}
\hline Simulation & $\alpha_{\mathrm{h} 0}$ & $\alpha_{\mathrm{h} 1}$ & $\alpha_{\mathrm{h} 2, \mathrm{en}}$ & $\alpha_{\mathrm{h} 2, \mathrm{ep}}$ & \multicolumn{1}{c}{$\alpha_{12}$} \\
\hline REF & $15^{\circ}$ & $-60^{\circ}$ & $-15^{\circ}$ & $-15^{\circ}$ & $0^{\circ}$ \\
TRANS & $15^{\circ}$ & $-60^{\circ}$ & $-15^{\circ}$ & $-15^{\circ}$ & $13.5^{\circ}$ \\
HELIX & $0^{\circ}$ & $-60^{\circ}$ & $0^{\circ}$ & $0^{\circ}$ & $0^{\circ}$ \\
\hline
\end{tabular}

